\title{
Splenic Palpation for the Evaluation of Morbidity due to Schistosomiasis Mansoni
}

\author{
Rogério Gerspacher-Lara, Rogério A Pinto-Silva, José Carlos Serufo, \\ Abdunnabi AM Rayes, Sandra C Drummond*, José Roberto Lambertucci/+
}

Faculdade de Medicina, Universidade Federal de Minas Gerais, Av. Alfredo Balena 190, 30130-100 Belo Horizonte, MG, Brasil *Fundação Nacional de Saúde, Belo Horizonte, MG, Brasil

This study was undertaken to determine the accuracy of splenic palpation for the diagnosis of splenomegaly, and to determine whether the frequency of individuals with a palpable spleen in an endemic area can be considered as an index of morbidity of schistosomiasis. For the clinical diagnosis of splenomegaly, two criteria have been tested: $(A)$ presence of a palpable spleen and $(B)$ presence of a palpable spleen whose border could be felt more than $4 \mathrm{~cm}$ below the costal margin. In an area of high prevalence of the disease (66.3\%) 285 individuals aged 18 years or more have been submitted to abdominal ultrasonography and physical examination. Splenomegaly was defined as a splenic length greater than $120 \mathrm{~mm}$ by ultrasound and the sensitivity, specificity, positive and negative predictive values of criterion A were $72.2 \%, 90.5 \%, 35.1 \%$ and $97.8 \%$. The values for criterion $B$ were $27.8 \%$, 98\%, $50 \%$ and $95 \%$, respectively. In an non endemic area, 517 individuals were submitted to the same protocol and 22 individuals had a palpable spleen, but no patient fulfilled criterion B for splenomegaly, and only one met the ultrasonographic criterion for splenomegaly. The authors concluded that abdominal palpation is a poor method for the diagnosis of splenomegaly.

Key words: schistosomiasis - spleen - abdominal palpation - ultrasound

Much of our knowledge about epidemiology and the natural history of schistosomiasis mansoni is derived from studies made in Africa and Latin America from the beginning of this century to the seventies. In these studies it was tried to determine the prevalence of the different forms of the disease, its relationship with gender, age or race of the individuals, and with other factors, like egg output, prevalence of the infection in the community and so on. In all these earlier studies, the only available mean to establish the diagnosis of the form of schistosomiasis was physical examination, probably a method of limited accuracy. In spite of that, results and conclusions of these pioneer works are still accepted and the prevalence of individuals with a palpable spleen among adolescents in endemic areas has been regarded as an index of morbidity due to schistosomiasis (Barreto et al. 1985). Today the general availability of portable ultrasound makes it possible to check the accuracy of clinical examination.

\footnotetext{
${ }^{+}$Corresponding author. Fax: $+55-31-273.4985$. E-mail: lamber@net.em.com.br Received 4 May 1998 Accepted 31 August 1998
}

A central point in most field studies has been the identification of individuals having hepatosplenic schistosomiasis. These individuals can be distinguished from others with hepatointestinal schistosomiasis basically by the presence of splenomegaly and this has been attempted by abdominal palpation.

In the last two decades some works comparing splenic palpation to more accurate means for the diagnosis of splenomegaly (scintilography and ultrasound) have been published (Westin et al. 1972, Sullivan \& Williams 1976, Barkun et al. 1991). Their results, however, can hardly be applied to field works, because they were performed in hospitals, where a substantial number of patients had massive splenomegaly, due to leukemia, policytemia vera, lymphoma or complicated portal hypertension, whereas in areas endemic for schistosomiasis, massive splenomegaly should not be expected to be so common. In these studies the sample was artificially composed, including very high numbers of individuals with splenomegaly, what makes calculation of predictive values for general use meaningless. Some conclusions of these studies, however, are important and valid: first, the vast majority of spleens of normal size are not palpable. It has also been established that some spleens of normal size are palpable (Sullivan $\&$ Williams 1976) and it was confirmed that many 
enlarged spleens are not palpable. If only 2 or $3 \%$ of the spleens of normal size are palpable, it is probable that the positive predictive value of the presence of a palpable spleen for the diagnosis of splenomegaly will be low in most communities because very few patients are likely to have diseases causing splenomegaly. In most textbooks of semiology or differential diagnosis, however, statements such as "a palpable spleen usually means splenomegaly" (Barondess \& Carpenter 1994) or even "the spleen must be enlarged two to four times to be palpable" (Schlossberg \& Shulman 1996) are still found.

The aim of this study was to determine the sensitivity, specificity, positive and negative predictive values of abdominal palpation for the diagnosis of splenomegaly, in the specific set of a field work in an area of high prevalence of schistosomiasis mansoni, and to compare the prevalence of splenomegaly, detected by ultrasonography and physical examination, in an endemic and in a control area, to determine if it can be considered as an index of morbidity of schistosomiasis mansoni.

\section{MATERIALS AND METHODS}

Studied areas - Queixadinha, a rural community in the district of Caraí, is located in the northeast of the State of Minas Gerais, Brazil, an area known to be highly endemic for schistosomiasis mansoni but where no cases of malaria or acute Chagas' disease were reported in recent years. In Queixadinha no cases of visceral leishmaniasis have ever been identified. It has been planned to submit all the population to two stool examinations. All adults and children older than five years would also be submitted to abdominal ultrasonography. More details about the study area can be found elsewhere (Lambertucci et al. 1996, Gerspacher-Lara et al. 1997).

The control area was Capão, another rural community in the district of Presidente Juscelino, in the center of the state. Although life standards were similar in both areas, for unknown reasons, transmission of schistosomiasis probably does not occur in Capão and other tropical diseases that can cause splenomegaly have never been identified there.

Patients - In the endemic area 551 individuals aged 0 to 86 years, $92 \%$ of the local population, have been submitted to physical examination and ultrasonography. All patients were submitted to two stool examinations by the Kato-Katz technique (Katz et al. 1972). Prevalence of the disease in this age group was $62 \%$. Abdominal palpation was performed with patients in dorsal decubit, during deep breath by two physicians, in a blinded fashion. Spleen has been considered to be palpable when splenic border could be felt below costal margin by both examiners. The greatest distance between splenic border and costal margin was also independently measured by the examiners. The second criterion tested was a distance between splenic border and costal margin (DSBCM) greater than $4 \mathrm{~cm}$. The latter had already been proposed as an attempt to improve the accuracy of the diagnosis of hepatosplenic schistosomiasis by physical examination (Prata 1987). Again, it was considered to be fulfilled if both observers registered distance greater than $4 \mathrm{~cm}$. Informed consent was obtained for this study.

In the control area 517 individuals (89\% of the total population) have been submitted to the same protocol of clinical, parasitological and ultrasonographic examinations. Only one individual that lived in a region known to be endemic for schistomiasis had eggs of $S$. mansoni in his faeces.

Ultrasonography - Abdominal ultrasonography was performed using real time equipment with electronic linear (Hitachi EUB 200) 3.5 MHz transducers. The splenic transverse diameter was recorded by an ultrasonographist who was unaware of the results of physical examination. Splenomegaly was considered to be present when splenic lenght was greater than $120 \mathrm{~mm}$, for individuals older than 17 years, the definition proposed by the World Health Organization for adults from Africa and Latin America (Hatz et al. 1992).

Because of the lack of widely accepted quantitative criteria for the ultrasonographic diagnosis of splenomegaly in children, we restricted our analysis of the specificity and sensitivity of the splenic palpation to individuals aged 18 years or more.

\section{RESULTS}

Physical examination versus ultrasonography in the endemic area - The spleen has been palpated by both physicians in 37 cases (discordance between examinators occurred in five cases). In 10 patients whose spleen had been palpated the distance between splenic and costal borders were considered to be greater than $4 \mathrm{~cm}$ by both examinators (discordance occurred in two cases).

Mean splenic length in patients with and without palpable spleen was 10.4 and $7.1 \mathrm{~cm}$ respectively $(\mathrm{p}<0.000001)$. Among patients with palpable spleens the mean length in patients that did and did not fulfill criterion B (DSBCM $>4 \mathrm{~cm}$ ) was $109.0 \mathrm{~mm}$ and $101.1 \mathrm{~mm}$, respectively $(\mathrm{p}=0.13)$.

The values of sensitivity, specificity, positive and negative predictive value for criterion A (palpable spleen) and B (DSBCM > 4cm) are shown in Table I. 
We compared the prevalence of splenomegaly defined by different criteria and in different age groups in the endemic and control areas (area I and II respectively). The results are shown in Table II.

\section{DISCUSSION}

The results of this work show that in spite of a relatively small interobserver variability, splenic palpation is a poor method for the diagnosis of splenomegaly.

If the presence of a palpable spleen is considered as a sign of splenic enlargement, in the endemic area its sensitivity would be relatively high, $(72.2 \%)$. The positive predictive value on the other hand was surprisingly low (35.1\%).

The second criterion tested for the diagnosis of splenomegaly, distance between splenic border and costal margin greater than $4 \mathrm{~cm}$, did not considerably improve the positive predictive value that remained as low as 50\% in the endemic area, using ultrasonography as gold standard, but reduced the sensitivity to $27.8 \%$. In the control area, on the other hand, no patient met this criterion, what suggests that it can be of some value for the evaluation of morbidity due to schistosomiasis, even if it greatly underestimates the number of patients with splenomegaly.

The fact that only one individual in the non endemic area had a splenic length greater than 120 $\mathrm{mm}$, strongly supports this sonographic definition of splenomegaly. The relatively frequent finding of individuals with a palpable spleen in this area, on the other hand, reinforces our conclusion that most patients with a palpable spleen do not have splenomegaly.

Probably the usage of an index of splenic volume calculated from the measurements of more than one splenic diameter would be a more accurate method for the ultrasonographic diagnosis and could have changed the results of this work, but it has been established in the literature that the longitudinal diameter is a good indicator of splenomegaly, and its use is firmly incorporated in the clinical practice.

Another conclusion from our data is that the frequency of individuals with a palpable spleen among adults is a better index of morbidity of schistosomiasis than the prevalence of palpaple spleens among children and adolescents, probably because many normal individuals aged less than 18 years have palpable spleen.

The limitations of the abdominal palpation for the diagnosis of splenomegaly are certainly due to the fact that many factors other than splenic size are important to determine if splenic border could be palpated. These factors probably include the thickness of abdominal wall, the position of the spleen, its shape and mobility and co-operation of the patient during physical examination. It is possible that such variables, that have never been adequately analyzed, have acted as confounders in previous works about schistosomiasis and other diseases causing splenomegaly.

\section{TABLE I}

Sensitivity, specificity, positive predictive value (PPV) and negative predictive value (NPV) of criterion A (palpable spleen) and B (distance between splenic border and costal margin $>4 \mathrm{~cm}$ ). Splenomegaly defined by splenic length $>120 \mathrm{~mm}$ by ultrasound

\begin{tabular}{ccccc}
\hline Criterion & Sensitivity & Specificity & PPV & NPV \\
\hline A & $13 / 18(72 \%)$ & $229 / 253(91 \%)$ & $13 / 37(35 \%)$ & $229 / 234(98 \%)$ \\
B & $5 / 18(28 \%)$ & $248 / 253(98 \%)$ & $5 / 10(50 \%)$ & $248 / 261(95 \%)$ \\
\hline
\end{tabular}

\section{TABLE II}

Comparison of prevalence of splenomegaly in the endemic and control area

\begin{tabular}{ccccc}
\hline Criterion/age & Prevalence area I & Prevalence area II & Odds ratio & $\mathrm{p}$ \\
\hline $\mathrm{A}$ & $18 / 71(7 \%)$ & $1 / 235(0 \%)$ & 15.6 & 0.0009 \\
$\mathrm{~B}$ & $37 / 271(14 \%)$ & $10 / 235(4 \%)$ & 3.2 & 0.002 \\
C & $22 / 280(8 \%)$ & $12 / 282(4 \%)$ & 1.8 & 0.13 \\
D & $10 / 271(4 \%)$ & $0 / 235(0 \%)$ & - & 0.0009 \\
\hline
\end{tabular}

Splenomegaly defined as: A - splenic length $>120 \mathrm{~mm}$ by ultrasound (individuals aged 18 years or more); B palpable spleen (individuals aged 18 years or more); C - palpable spleen (individuals aged 17 years or less); D palpable spleen and distance of the splenic border to costal margin greater than $4 \mathrm{~cm}$ (individuals aged 18 or more). 


\section{REFERENCES}

Barkun AN, Camus M, Green L, Meagher T, Coupal L, De Stempel J, Grover SA 1991. The bedside assessment of splenic enlargement. Am J Med 91: 512518.

Barondess JA, Carpenter CCJ 1994. Differential Diagnosis, Lea and Febiger, Malvern, Pennsylvania, 993 pp.

Barreto ML, Loureiro S, Melo MS, Anjos CFD 1985. The effect of Schistosoma mansoni infection on child morbidity in the State of Bahia, Brazil. II Analysis at the individual levell. Rev Inst Med Trop S Paulo 27: 167-171.

Gerspacher-Lara R, Pinto-Silva RA, Rayes AAM, Drummond SC, Lambertucci JR 1997. Ultrasonography of periportal fibrosis in schistosomiasis mansoni in Brazil. Trans $R$ Soc Trop Med Hyg 91: 307-309.

Hatz C, Jenkins JM, Morrow RH, Tamer M 1992. Ultrasound in schistosomiasis. A critical look at methodological issues and potential applications Acta Tropica 51: 89-97.
Katz N, Chaves AM, Pellegrino P 1972. A simple device for quantitative stool thick-smear technique in schistosomiasis mansoni. Rev Inst Med Trop S Paulo 14: 397-400.

Lambertucci JR, Gerspacher-Lara R, Pinto-Silva RA, Barbosa MM, Teixeira R, Barbosa HF, Serufo JC, Rezende DF, Rayes AAM 1996. O projeto Queixadinha: a morbidade o controle da esquistossomose em área endêmica no nordeste de Minas Gerais, Brasil. Rev Soc Bras Med Trop 29: 127-135.

Prata A 1987. Schistosomiasis mansoni in Brazil. In Mahmoud Schistosomiasis. Baillière's Clinical Tropical Medicine and Communicable Diseases 2: 349-369.

Schlossberg D, Shulman JA 1996. Differential Diagnosis of Infectious Diseases, 2nd ed., Williams \& Wilkins, Philadelphia, 260 pp.

Sullivan S, Williams R 1976. Reliability of clinical techniques for detecting splenic enlargement. Brit Med J 2: 1043-1044.

Westin J, Lanner LO, Larson A, Weinfeld A 1972. Spleen size in polycitemia. A clinical and scintigraphic study. Acta Med Scand 191: 263-271. 